\title{
New precision measurements of the strong interaction in kaonic hydrogen*
}

M. Bazzi ${ }^{1}$, G. Beer ${ }^{3}$, L. Bombelli ${ }^{1}$, A. M. Bragadireanu ${ }^{5}$, M. Cargnelli ${ }^{2}$, M. Catitti ${ }^{1}$, C. Curceanu (Petrascu) ${ }^{1,5}$, C. Fiorini ${ }^{4}$, T. Frizzi ${ }^{4}$, F. Ghio ${ }^{6}$, B. Girolami ${ }^{6}$, C. Guaraldo ${ }^{1}$, M. Iliescu ${ }^{1}$, T. Ishiwatari ${ }^{2}$, P. Kienle ${ }^{2, * *}$, P. Lechner ${ }^{7}$, J. Marton ${ }^{2} * * *$, K. Nikolics ${ }^{2}$, P. Levi Sandri ${ }^{1}$, A. Longoni ${ }^{4}$, V. Lucherini ${ }^{1}$, D. Pietreanu ${ }^{1}$, T. Ponta ${ }^{5}$, D. L. Sirghi ${ }^{1}$, F. Sirghi ${ }^{1}$, H. Soltau ${ }^{1}$, L. Strüder ${ }^{8}$, O. Vazquez Doce ${ }^{1}$, E. Widmann ${ }^{2}$, J. Zmeskal $^{2}$

1 Laboratori Nazionali di Frascati, INFN, Frascati, Italy

2 Stefan-Meyer-Institut, Austrian Academy of Sciences, Vienna, Austria

3 Dept. of Physics and Astronomy, Univ. of Victoria, Victoria, Canada

4 Dip. di Elettronica, Politecnico di Milano, Milano, Italy

5 IFIN-HH, Bucharest, Romania

6 INFN, Sez. di Roma I and Instituto Superiore di Sanità, Rome, Italy

7 PNSensors GmbH, Munich, Germany

8 MPI for Extraterrestrial Physics, Garching, Germany

Received 29 November 2007; Accepted 11 May 2008; Published online 19 November 2008 (C) Springer-Verlag 2008

\begin{abstract}
The DEAR experiment performed at the DA $\Phi$ NE electron-positron collider (Frascati, Italy) studied the strong interaction shift and width of the $1 \mathrm{~s}$ state in kaonic hydrogen using X-ray spectroscopy. The repulsive character of the kaon-proton interaction at threshold was confirmed and the most precise values of the shift and width were obtained. However, high precision data at the percent level are highly requested to further develop the theories and thus our understanding of the low-energy antikaon-nucleon interaction. Therefore, a new series of precision experiments on kaonic hydrogen and kaonic deuterium are planned at LNF.
\end{abstract}

\footnotetext{
* Presented at the 20th Few-Body Conference, Pisa, Italy, 10-14 September 2007

** Alternative address: TU Munich

*** E-mail address: johann.marton@oeaw.ac.at
}

Correspondence: J. Marton, Stefan-Meyer-Institut, Austrian Academy of Sciences, Boltzmanngasse 3, 1090 Wien, Austria 


\section{Introduction}

The low-energy domain of the antikaon $(\bar{K})$-nucleon interaction is neither simple nor well understood up to now. The precise measurement of the X-ray spectrum of kaonic atoms can be regarded as a window to study the $\bar{K} N$ interaction at threshold. Since only the $1 s$ state of kaonic hydrogen and deuterium are influenced by strong $\bar{K} N$ interaction, the measurement of the energy of the Lyman X-ray transition and subtracting the calculated electromagnetic value $E_{n p \rightarrow 1 s}^{\text {e.m.,calc. }}$

$$
\epsilon_{1 s}=E_{n p \rightarrow 1 s}^{\text {meas. }}-E_{n p \rightarrow 1 s}^{\text {e.m.,calc. }}
$$

leads to the shift $\epsilon_{1 s}$. The width $\Gamma_{1 s}$ manifests in the broadening due to the reduced lifetime. From these observables the low energy parameters, the scattering lengths, can be extracted applying Deser-type formulae [1] in a fairly direct way. In first order neglecting isospin breaking the complex kaon-proton scattering length $a_{K^{-}}$is given by the following formula (a similar formula holds true for $a_{K^{-}}$)

$$
\epsilon_{1 s}+i \frac{\Gamma_{1 s}}{2}=2 \alpha^{3} \mu^{2} a_{K^{-} p}=412 \cdot \mathrm{fm}^{-1} \cdot \mathrm{eV} \cdot a_{K^{-} p} .
$$

The values of the scattering amplitudes at threshold can be easily compared with theoretical results thus testing theory in a rather direct and accurate way. New experiments at LNF planned by the SIDDHARTA collaboration [2] will combine the advantages of DA $\Phi$ NE as a source of monoenergetic $K^{-}$with low energy and new state-of-the-art X-ray detectors.

\section{Exotic atom research at DA $\Phi$ NE-DEAR}

The experimental program of DEAR was successfully completed: For the first time data on X-ray transitions in kaonic nitrogen were studied in detail [3]. These data provide a valuable input for cascade calculations in exotic atoms. Most important are the new data on $\epsilon_{1 s}$ and $\Gamma_{1 s}$ of kaonic hydrogen [5]. The repulsive character of the $\bar{K} N$ interaction at threshold found in the pioneering $\mathrm{KpX}$ experiment [4] was verified. However, smaller values for $\epsilon_{1 s}$ and $\Gamma_{1 s}$ were found at the highest accuracy up to now. The precision of the DEAR experiment was limited due to the high soft X-ray background. This limitation will be overcome in the new SIDDHARTA experiment.

\section{The SIDDHARTA experiment}

The physics goal of the SIDDHARTA (silicon drift detectors for hadronic atom research using timing application) are precision measurements (i.e., at the $\mathrm{eV}$ level) of the X-ray spectra of kaonic hydrogen and - for the first time - kaonic deuterium. This will lead to a breakthrough in the field of low-energy $\bar{K} N$ interaction. The main features of our experiment at the DA $\Phi N E$ facility are:

(1) New X-ray detectors with timing capability for reducing the soft X-ray background by using a triple coincidence $K^{-} \times K^{+} \times X$.

(2) Nearly mono-energetic $K^{-}$from $\Phi$-meson decay $\left(E_{K^{-}} \sim 16 \mathrm{MeV}\right)$.

(3) Increased luminosity at DA $\Phi N E$. 
The R\&D of the large area $\left(1 \mathrm{~cm}^{2}\right.$ active area) SDDs [6] and the associated readout electronics is in the final stage. The spectroscopic tests of the SDDs show an excellent energy resolution of $\sim 135 \mathrm{eV}$ at $6 \mathrm{keV}$ corresponding to the $K_{\alpha}$ transition energy. The timing performance was checked to be appropriate to the requirements. At this point it has to be stressed that large area SDDs (a type with external preamplifier) were already used successfully in an experiment at KEK on the measurement of the Balmer series of kaonic ${ }^{4} \mathrm{He}[7]$.

\section{Outlook}

As a first step a reduced setup will be used to examine the performance of the apparatus and to study the background condition. After this initial phase the final setup of SIDDHARTA will be installed applying an array of $\sim 150$ SDDs. Examples of the expected X-ray spectrum of kaonic deuterium for $600 \mathrm{pb}^{-1}$ are given in Fig. 1 (assuming $\epsilon_{1 s}=662 \mathrm{eV}$ and $\Gamma_{1 s}=1209 \mathrm{eV}$ [8] (top), $\epsilon_{1 s}=325 \mathrm{eV}$ and $\Gamma_{1 s}=$ $630 \mathrm{eV}$ [9] (bottom)). The precision in $\epsilon_{1 s}$ and $\Gamma_{1 s}$ is well below $10 \%$.

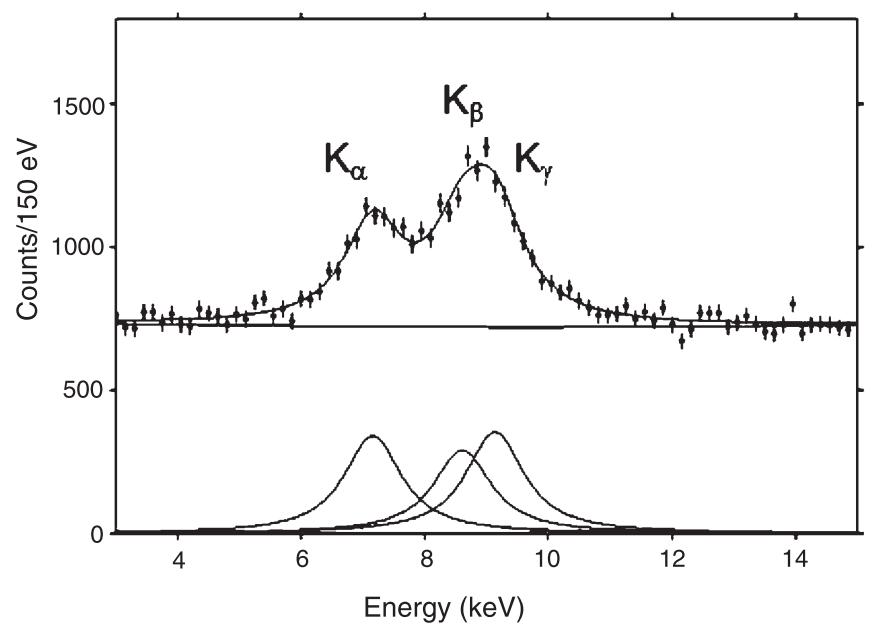

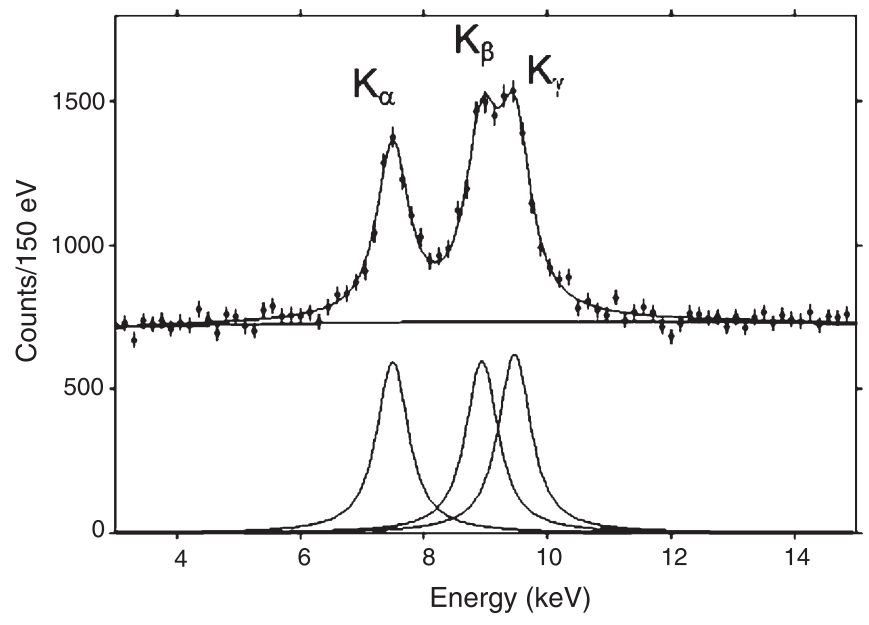

Fig. 1 Monte Carlo simulated Xray spectra of kaonic deuterium with yield of $K_{\alpha}=0.2 \%$ 
Acknowledgement This work was partly supported by EU within I3-HadronPhysics (Joint Research Activity 10), Austrian Federal Ministry of Science and Research, and TARI-INFN, Contract No. RII3CT-2004-506078.

\section{References}

1. Deser S, et al. (1954) Phys Rev 96:774

2. http://www.lnf.infn.it/esperimenti/siddharta/, Curceanu C, et al. (2007) Proc. HYP06:189

3. Ishiwatari T, et al. (2004) Phys Lett B 593:48

4. Iwasaki M, et al. (1997) Phys Rev Lett 78:3067

5. Beer G, et al. (2005) Phys Rev Lett 94:212302

6. Ishiwatari T (2007) Nucl Instr Meth A 581:326

7. Okada S, et al. (2007) Phys Lett B 653:387

8. Meißner U-G, Raha U, Rusetsky A (2006) Eur Phys J C 47:473

9. Ivanov AN, et al. (2005) Eur Phys J A 23:79 